\title{
René-Charles Guilbert de Pixerécourt, Mélodrames
}

\section{Valentina Ponzetto}

\section{(2) OpenEdition}

\section{Journals}

\section{Édition électronique}

URL : https://journals.openedition.org/studifrancesi/21351

DOI : 10.4000/studifrancesi.21351

ISSN : 2427-5856

\section{Éditeur}

Rosenberg \& Sellier

\section{Édition imprimée}

Date de publication : 1 décembre 2019

Pagination : 580-581

ISSN : 0039-2944

\section{Référence électronique}

Valentina Ponzetto, «René-Charles Guilbert de Pixerécourt, Mélodrames », Studi Francesi [En ligne], 189 (LXIII | III) | 2019, mis en ligne le 01 mars 2020, consulté le 11 novembre 2021. URL : http:// journals.openedition.org/studifrancesi/21351 ; DOI : https://doi.org/10.4000/studifrancesi.21351

Ce document a été généré automatiquement le 11 novembre 2021.

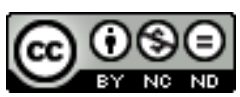

Studi Francesi è distribuita con Licenza Creative Commons Attribuzione - Non commerciale - Non opere derivate 4.0 Internazionale. 


\title{
René-Charles Guilbert de Pixerécourt, Mélodrames
}

\author{
Valentina Ponzetto
}

\section{RÉFÉRENCE}

René-Charles Guilbert de Pixerécourt, Mélodrames, dir. R. Martin, t. III - 1804-1808, édition critique par K. Astbury, B.T. Cooper, R. Martin et S. Robardey-Eppstein, Paris, Classiques Garnier, 2016, $1234 \mathrm{pp}$.

1 Après un premier et un deuxième volume (parus respectivement en 2013, v. "Studi Francesi" 176 et en 2014, v. "Studi Francesi" 183) se poursuit la monumentale édition des Mélodrames de Pixerécourt sous la direction de Roxane Martin, première édition intégrale et scientifique de cet auteur aujourd'hui oublié qui eut toutefois une si grande importance pour la formation des goûts théâtraux de toute une génération et une influence désormais en cours de pleine réévaluation sur le drame romantique.

2 Les principes éditoriaux restent les mêmes que pour les volumes précédents. Les textes sont établis à partir du manuscrit autographe et, quand les documents conservés le permettent, à partir du manuscrit soumis à la censure. Certaines pièces sont enrichies de la partition musicale composée pour la création parisienne, quand les documents conservés permettent de la reconstruire. Toutes les pièces sont précédées d'une présentation précisant le contexte d'écriture et de représentation. Le volume dans son ensemble est accompagné d'une riche bibliographie et d'une chronologie détaillée pour la période concernée.

3 Ce troisième volume couvre les années 1804-1808, époque de grands bouleversements politiques qui suivent la proclamation du Premier Empire et accompagnent les guerres napoléoniennes, avec leurs jeux d'alliances et de conflits successifs. Ces aléas de l'actualité, rappelle Roxane Martin dans l'«Avant-Propos» général du volume, se traduisent dans l'écriture des mélodrames, témoignant «le positionnement politicoaffectif de l'auteur par rapport aux événements contemporains» (p.10). Les six 
mélodrames de cette période rencontrent moins de succès que les précédents, d'un côté pour le traitement distancié et imprégné de références littéraires des thématiques militaires, de l'autre à cause des décrets sur les théâtres de 1807, qui rétablissant le système des privilèges et ordonnant la fermeture de bon nombre de théâtres privés privent Pixérécourt de l'une des scènes les plus appréciées du public de l'époque: ce théâtre de la Porte-Saint-Martin où il a connu ses meilleurs succès.

4 Sylviane Robardey-Eppstein présente Les Maures d'Espagne, ou Le Pouvoir de l'enfance (pp. 27-151), mettant en scène la couleur locale d'une péninsule ibérique au temps de l'invasion arabe, et plus particulièrement de la chute de Grenade. Dans cette pièce «la thématique et la morale du pouvoir de l'enfance et de ses larmes» (p. 55), à forte valeur pathétique et sentimentale est centrale, et connaît son point d'orgue dans l'épisode des enfants envoyés plaider dans le camp ennemi. Toutefois le mélodrame sentimental ne peut pleinement réussir que grâce aux pouvoirs visuels d'une conception scénique à grand spectacle, à grand renfort de chants, ballets, musique, scénographie ingénieusement étudiée et d'une bonne dose d'exotisme. Aux sources de l'intrigue on trouve en même temps un roman de Florian de sujet mauresque et une pièce de sujet allemand de Kotzebue, Les Hussites, dont Duval avait tiré presque en même temps un autre mélodrame pour la Porte-Saint-Martin. Cela valut à Pixerécourt une âpre polémique dans la presse du temps, minutieusement reconstruite dans la présentation.

5 Le Grand chasseur, ou L'île des palmiers (pp. 153-262), écrit en collaboration avec Loaisel de Tréogate dans des proportions que Roxane Martin, dans sa présentation, s'efforce de quantifier et de justifier, exploite un matériel historique semblable, renvoyant à l'invasion de l'Espagne par les Maures et aux romances du Roi Rodrigue, mais en complexifiant les données historico-légendaires par des échos classiques (notamment avec l'Énéide) et bibliques. L'amour-passion y est représenté dans son articulation avec la guerre et dans ses conséquences sur le destin des peuples. L'ensemble, où le mélange entre registre tragique et mélodramatique, entre amour blessé et enjeux politiques a du mal à fonctionner, fut perçu par les contemporains comme «un alliage malhabile entre ingrédients jugés disparates» (p. 170) et dérouta les spectateurs qui, n'y retrouvant pas leurs codes dramaturgiques habituels et favoris, en déterminèrent la chute retentissante.

6 Encore adaptée de Kotzebue, La Forteresse du Danube (pp. 262-411) transpose le Hugo Grotius de l'auteur allemand, drame sur le thème de la persécution religieuse et de la liberté de conscience, en gommant les personnages historiques de Grotius et du prince d'Orange qui le fit emprisonner et les remplaçant par un Evrard de fantaisie et par Joseph II d'Autriche. Katherine Astbury étudie de près les modifications apportées par Pixerécourt, notamment pour diminuer l'importance de l'intrigue amoureuse et pour éviter toute allusion possible à Napoléon ou à l'Empire, et les particularités dramaturgiques de la pièce, qui obtint un succès durable à Paris comme en province et même à l'étranger. Ce mélodrame fut créé en janvier 1805 au théâtre de la Porte-SaintMartin. Le choix du théatre et de sa troupe impliquait comme conséquences une place plus significative accordée au ballet, qui déterminera d'ailleurs le succès de la pièce, et une plus grande importance des personnages comiques. L'alternance du pathétique et du comique deviendra ainsi à partir de 1805 l'une des marques distinctives des mélodrames de Pixerécourt, si bien qu'on peut considérer La Forteresse du Danube un tournant dans sa carrière. 
7 Robinson Crusoé (pp. 413-586) pose un défi différent: adapter un roman très connu, qu'il est impossible de porter à la scène tel quel par son éclatement spatio-temporel, mais dont il est impératif de garder intacts les moments et les éléments les plus reconnaissables, sous peine de décevoir les attentes des spectateurs. Barbara Cooper analyse le processus d'adaptation et les enjeux des changements majeurs opérés par Pixerécourt, notamment pour resserrer l'intrigue dans les trois unités imposées par la dramaturgie classique et pour introduire d'un côté l'élément spectaculaire des mœurs des «sauvages» (s'inspirant en partie, de son propre aveu, des Mours des sauvages américains du père Lafitau), de l'autre l'élément pathétique et sentimental lié à l'introduction de nombreux personnages ayant des liens de parenté avec Robinson ou Vendredi. Le succès de public fut au rendez-vous, comme le montrent la revue de presse de la création, les parodies qui fleurirent immédiatement (Robinson cadet; GillesRobinson et Arlequin-Vendredi) et les reprises successives en France et à l'étranger.

Un fait historique autour d'un conflit de succession et de partage de biens entre seigneurs de Montfaucon et comtes de Neuchâtel et de Grandson au XIV siècle, déjà mis en scène par Kotzebue dans Johanna von Montfaucon, est à l'origine du Solitaire de la roche noire (pp.587-712), et offre à Pixerécourt l'occasion de questionner les liens entre pouvoir légitime et héréditaire. À travers l'analyse des changements apportés par Pixerécourt à l'original allemand, Roxane Martin fait émerger les motifs chers au dramaturge français, par exemple que «la légitimité s'acquiert grâce à l'exercice de la vertu» (p. 605), ou que le sentiment apparaît en fin de compte «comme la seule valeur susceptible de fonder un jugement» (p.607) dans un monde au partage manichéen où les personnages se définissent par leur posture morale, faisant glisser la pièce, malgré ses personnages féodaux du Moyen Âge, vers un registre de mélodrame bourgeois.

Sylviane Robardey-Eppstein met enfin à jour les sources d'inspiration possibles et croisées, entre Schiller, Klingemann et des œuvres précédentes du même Pixerécourt, de L'Ange tutélaire, ou Le Démon femelle (pp. 713-1030), sombre histoire de conspiration doublée d'une trahison entre frères dans l'Italie du $\mathrm{xvI}^{\mathrm{e}}$ siècle. Derrière l'anecdote éloignée dans le temps et l'espace d'une Ferrare de la Renaissance S. Robardey-Eppstein lit pourtant un palimpseste d'allusions à la politique contemporaine de la famille Bonaparte, Napoléon, mais aussi son frère Joseph au royaume de Naples, laissant lire le mélodrame comme une réflexion de philosophie politique. Des analyses intéressantes sont consacrées aussi aux personnages les plus originaux et subversifs de la pièce, l'héroïne Flora, femme rebelle et combative, à contre-courant des figures féminines habituelles du mélodrame, et une paire de bandits grotesques et sympathiques, inversant de leur côté les stéréotypes du personnage négatif. Cas unique dans ce volume III, la pièce est accompagnée de la partition d'orchestre originale établie par Clara Rico Osés. 